\title{
Cilium-generated signaling and cilia-related disorders
}

\author{
Junmin Pan, Qian Wang and William J Snell \\ Department of Cell Biology, University of Texas Southwestern Medical School, Dallas, TX, USA
}

\begin{abstract}
Biologists have long known that humans experience their environment through cilia. Light, odorant, and sound perception depend on these microtubule-filled, complex organelles present on cells in primary sensory tissues. Recently, discoveries on the mechanism of assembly of cilia (flagella) in the lowly, biflagellated, eucaryotic green alga Chlamydomonas have triggered a renaissance of interest in the organelles along with a recognition of their key sensory roles in nonsensory tissues. Chlamydomonas researchers uncovered an entirely new set of cellular machinery essential for transporting the protein components of cilia and flagella in all ciliated/ flagellated eukaryotic cells between their site of synthesis in the cell body and their site of assembly at the tip of the flagellum (intraflagellar transport: IFT). Prompted by the surprising observations that disruption of IFT genes in mice led to polycystic kidney disease (PKD) and that PKD proteins are present on the sensory cilia of Caenorhabditis elegans, researchers have made a direct connection between PKD and cilia. At least five (and possibly all) of the seven identified human genes disrupted in PKD and a related disorder nephronophthisis encode proteins expressed in the primary cilia that project into the lumen from the epithelial cells that line renal tubules. Moreover, the renal cilia are flow sensors and at least two of the PKD genes encode ciliary transmembrane proteins essential for mechanosensation. Although their roles have not yet been as clearly identified, cilia also are at the center of a rare human disorder, Bardet-Biedl syndrome (BBS), in which patients exhibit phenotypes of common human diseases, including obesity and increased incidence of hypertension and diabetes. Five of the eight known BBS genes encode basal body or cilia proteins in mice or humans, and homologues of two of the remaining genes are present in basal bodies/cilia of model organisms. Here we briefly describe the biology of cilia and flagella, we outline how studies on model organisms have led to our current understanding of the roles of these organelles and their proteins in health and disease, and we highlight the notion that the primary cilia present on cells throughout the body, even those on brain neurons, may be essential for as yet undiscovered cilium-generated signaling functions.

Laboratory Investigation (2005) 85, 452-463, advance online publication, 21 February 2005; doi:10.1038/labinvest.3700253
\end{abstract}

Keywords: Chlamydomonas; cilia; flagella; intraflagellar transport

\section{A cilium on (almost) every cell}

Unbeknown to most biomedical scientists, cilia and flagella are present on almost all human cells, including cells in the embryo (see the following URL for a comprehensive list of where primary cilia are found: http://members.global2000.net/bowser/ cilialist.html). Figure 1 presents images showing cilia on chondrocytes (Figure 1a), brain neurons (Figure 1b), and renal epithelial cells (Figure 1c). Cilia and flagella are microtubule-filled, cellular extensions whose enclosing membrane is continu-

Correspondence: Dr WJ Snell, PhD, Department of Cell Biology, University of Texas Southwestern Medical School, Rm. K2-226, 5323 Harry Hines Blvd., Dallas, TX 75390-9039, USA.

E-mail: william.snell@utsouthwestern.edu

Received and accepted 16 December 2004; published online 21 February 2005 ous with the cell plasma membrane. Although cilia and flagella are identical in structure and composition, the two names were originally coined to indicate distinctive patterns of movement and are still used. Flagella show symmetrical undulations that pass along the length of the organelle, whereas motile cilia show a more eccentric beat, characterized by a bend at the base during the power stroke followed by a recovery stroke as a bent organelle. Medical students are most familiar with the multiple, motile cilia on the cells that line the lumens of ducts in several tissues, but many cilia are nonmotile and are present just one per cell. ${ }^{1-3}$

Cilia play obvious roles in several tissues, including cell motility (sperm) and transport of mucus, other fluids, and even other cells (oviduct and efferent ducts of the testis). Less well appreciated are the roles of cilia in allowing us to experience our environment. We see, hear, and smell with cilia. 

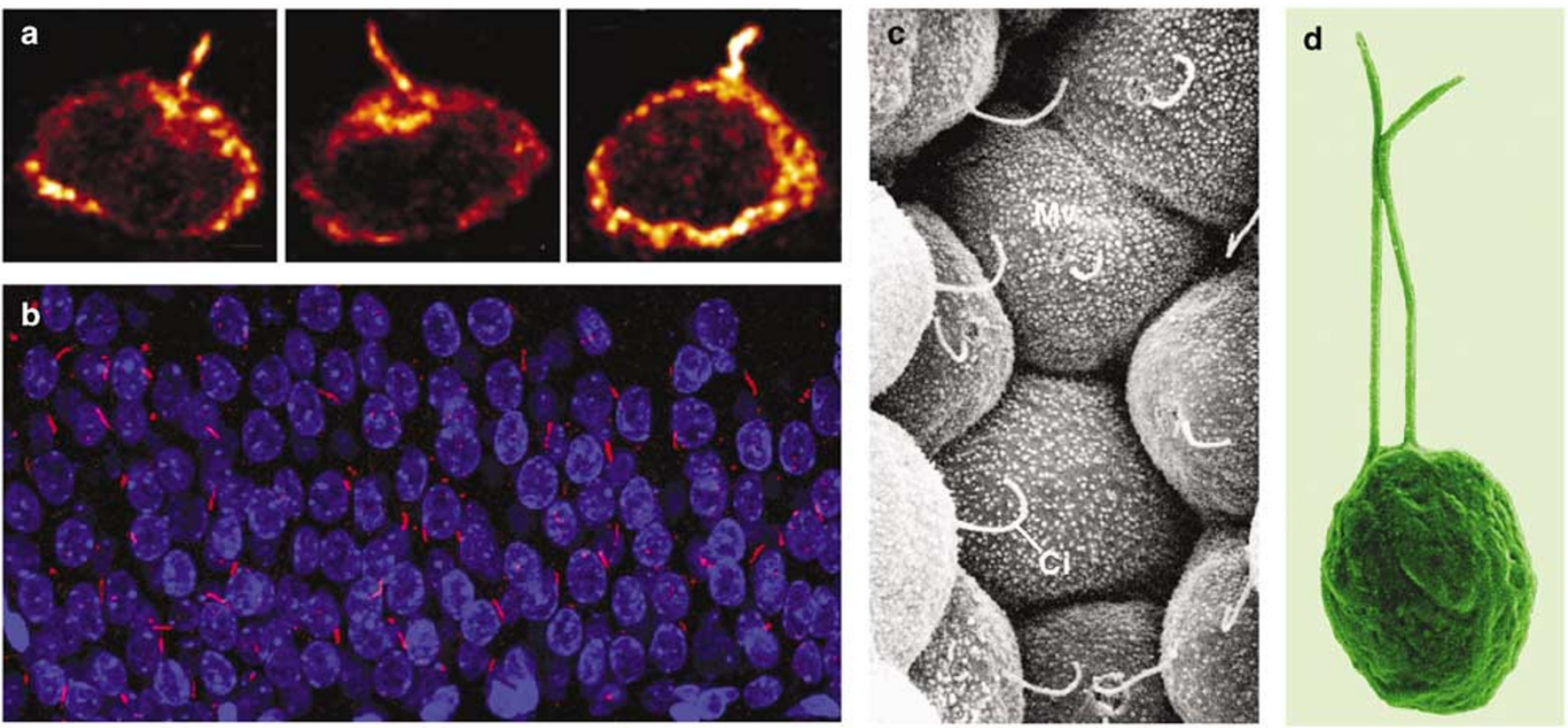

Figure 1 Examples of cells with cilia or flagella. (a) Immunofluorescence images of three chondrocytes from chick embryo sterna showing primary cilia stained for acetylated alpha-tubulin. Primary cilia project into the unstained hyaline cartilage extracellular matrix and display a range of ciliary bending patterns. This range of bending patterns is thought to be mediated through interactions with collagen fibers (see Jensen et $\mathrm{al}^{112}$ ). The image was generously provided by SR McGlashan, CA Poole, and CG Jensen of the University of Auckland, Auckland, New Zealand. (b) Primary cilia of the granule cells in the granule cell layer of rat hippocampus stained with somatostatin receptor-three antibody (red); nuclei are counterstained with Hoecsht dye (blue). The image was kindly provided by SR McGlashan, MYF Kam, CA Poole, and CG Jensen of the University of Auckland, Auckland, New Zealand. (c) Scanning electron micrograph of renal epithelial cells in a collecting tubule of the kidney. Each cell has a primary cilium. (Taken from Kessel and Kardon ${ }^{113}$ and used with permission of Randy $\mathrm{H}$ Kardon.) (d) Scanning electron micrograph of the unicellular, biflagellated green alga Chlamydomonas reinhardtii. The image was kindly provided by Dr William Dentler, University of Kansas, Lawrence, KS.

The photoreceptor proteins in the vertebrate retina are housed in a modified, nonmotile cilium, the rod outer segment. Odorant receptors of olfactory epithelial cells are expressed on the tufts of long cilia that project from the olfactory knob. And the single kinocilium on the hair cells of the ear is intimately associated with the stereocilia (actinfilled microvilli), whose movements with respect to each other lead to sound perception. In fact, a putative mechanosensory channel protein in hair cells is more abundant in the kinocilium than in the surrounding stereocilia. ${ }^{4}$ Recent studies have led to a much fuller appreciation of the fact that not only do cilia act in sensory roles at critical stages in embryonic development, but their sensory roles are essential for the normal functioning of many tissues, including the kidney! Moreover, new studies on a rare human disease characterized by common disorders (obesity, diabetes, hypertension) raise the possibility that cilia play roles in energy metabolism and regulation of blood pressure.

Here, we present an overview of our current knowledge of cilia and flagella and their roles in human disease. (Table 1 presents an overview of the locations and properties of cilia that are known to be associated with several human phenotypes.) We will begin with a brief description of the structural and motile properties of cilia and flagella, summarizing how that information previously led to an understanding of several human diseases such as Kartagener's syndrome. We will then outline how recent studies on the biflagellated green alga Chlamydomonas (Figure 1d) and other model organisms stimulated entirely new areas of research on cilia, and we will briefly summarize our updated understanding of the defects in cilia that underlie several clinically important, 'cilia-related disorders' ${ }^{5,6}$ As we hope will become clear to the reader, a consideration only of the genes that encode structural and motor proteins of cilia goes just partway toward a full understanding of what can go wrong with these organelles. Discovery of new, cilia-related genes that cause human disease came from studies of the mechanism of assembly of the organelles. Out of those discoveries emerged the recognition that membrane proteins involved in human disease have unique functions on cilia. We will end the review with a short discussion of new studies that hint that the assembly machinery itself might be one of the distinctive properties of cilia that have led to their being exploited during evolution for cilium-generated signaling.

\section{The structure and composition of cilia and a link to cell division}

A motile cilium contains nine sets of doublet microtubules arranged in nine-fold symmetry in 
Table 1 Cilia-related phenotypes

\begin{tabular}{|c|c|c|c|}
\hline Location of cilia & Properties/functions & Phenotype & Ciliary defect \\
\hline Embryonic node & $\begin{array}{l}\text { 9+0; motile and nonmotile/ } \\
\text { nonmotile cilia sense leftward } \\
\text { nodal flow created by motile cilia }\end{array}$ & Situs inversus & Immotility \\
\hline Ependymal cells in brain ventricles & $\begin{array}{l}9+2 ; \text { motile/flow of cerebrospinal } \\
\text { fluid }\end{array}$ & Hydrocephalus & Immotility \\
\hline Photoreceptor cells in retina & 9+0; nonmotile/light perception & Blindness & IFT? \\
\hline Apical knob on olfactory neurons & $\begin{array}{l}9+2 \text { (at the base, microtubule } \\
\text { singlets distally); nonmotile }\end{array}$ & Anosmia & No cilia \\
\hline $\begin{array}{l}\text { Epithelium of the upper and lower } \\
\text { airways }\end{array}$ & $9+2 ;$ motile/mucus clearance & $\begin{array}{l}\text { Respiratory tract infections, rhinitis, } \\
\text { sinusitis }\end{array}$ & Immotility \\
\hline Sperm & 9+2; motile/sperm motility & Male infertility & Immotility \\
\hline Oviduct epithelium & 9+2; motile/egg transport & Female infertility & Immotility \\
\hline $\begin{array}{l}\text { Epithelium of the kidney, bile, and } \\
\text { pancreatic ducts }\end{array}$ & 9+0; nonmotile/mechanical sensing & Cyst formation & Failure to signal \\
\hline Unknown & $\begin{array}{l}9+0 \text { ?; nonmotile?/function in } \\
\text { development and metabolism? }\end{array}$ & $\begin{array}{l}\text { Obesity, polydactyly, diabetes, } \\
\text { cognitive impairment, other } \\
\text { ailments }\end{array}$ & Failure to signal? \\
\hline
\end{tabular}

the form of a hollow cylinder that surrounds a central pair of singlet microtubules. The outer doublet microtubules contain motor complexes, the inner and outer dynein arms, which provide the ATP-hydrolysis-driven mechanical movement that causes adjacent microtubule doublets to slide past each other. Spoke-like structures (the radial spokes) project from each outer doublet toward the central pair. All of these microtubules and their associated proteins, which together compose the axoneme, are the core of the cilium. The axoneme serves as the internal framework that supports the cilium and provides a structure on which mechanical movement is generated. Exquisite coordination of the action of the dynein arms, in part regulated by the radial spokes, brings about the characteristic bending of the organelles. The outer and inner dynein arms and the radial spokes are large protein complexes each comprising 10-20 polypeptides. Among the many nonmotile cilia found on cells throughout the body, some may be missing the central pair of microtubules or the central pair and the dynein arms. In other modified cilia (eg, rod outer segments, cilia on olfactory epithelial cells), the outer microtubules may not always be doublets and fewer than nine might be present. Along with the tubulin proteins that make up the singlet and doublet microtubules, the structural proteins that compose cilia and flagella are highly conserved across species. Much of what we know about the structure and function of cilia and flagella comes from studies of the flagella of the unicellular, biflagellated green alga, Chlamydomonas (see review by Silflow and Lefebvre ${ }^{7}$.

One fascinating feature of cilia and flagella is that the basal body, which templates the assembly of their axonemes, contains the same organelle, the centriole, which (at least for vertebrates and higher animals) is also the defining element of another microtubule-related organelle, the centrosome. ${ }^{8}$ Centrosomes, which contain a pair of centrioles, are the organizing centers for cytoplasmic microtubules in the interphase cell and for the spindle microtubules in mitotic cells. The centriole is a hollow cylinder composed of nine sets of triplet microtubules arranged in nine-fold symmetry. In its role as specifier/organizer of cilia and flagella, the centriole is anchored to the plasma membrane through a collection of fibers, filaments, and dense material and along with the transition zone forms the basal body of the cilium/flagellum. The outer doublet microtubules of the axoneme are continuous with and built directly upon the inner two microtubules of the triplets of the centriole (for a recent review, see Beisson and Wright ${ }^{9}$ ).

In its role as an essential element of the centrosome in interphase and mitotic cells, neither of the centrioles in the pair nucleates microtubules directly from its triplet microtubules. Instead, the cytoplasmic microtubules are initiated from proteins within a cloud of pericentriolar material in which the centrioles are embedded. Not surprisingly, many of the protein components of the pericentriolar cloud are also present in the basal body complex, including centrin and several isoforms of tubulin..$^{9,10}$ As a further indication of the intimate link between basal bodies and centrosomes, in cells that have only a single cilium, the organelle grows from one of the two centrioles in the centrosome! ${ }^{2}$ A dual role of one of the centrioles means that during cell division, a process that requires both centrioles, the cilium must be disassembled to free its centriole for other purposes. As will be further illuminated below, the interrelationship between cilia, centrosomes, and cell division may be important in the cellular overproliferation that occurs in some cilia-related disorders. 


\section{Human diseases related to defects in motile cilia: primary ciliary dyskinesia}

One of the first diseases attributed to cilia, named Kartagener's syndrome, was associated with the triad of sinusitis, bronchitis, and situs inversus. ${ }^{11-13}$ Afzelius made the connection between the syndrome and cilia when he reported that many infertile males he studied had immotile cilia and also displayed Kartagener's triad. He also postulated in the 1976 paper that situs inversus might be due to defects in the cilia on embryonic epithelial cells whose normal ' . . . beating somehow is instrumental in determining the visceral situs'. ${ }^{11}$ The immotile cilia syndrome, also called primary ciliary dyskinesia (PCD),${ }^{14}$ is characterized by defects in structural/ motor components of cilia, including the dynein arms and radial spokes. Situs inversus, the condition in which the normal left-right symmetry of the internal organs is disrupted, is due to defective motility of cilia in a region of the embryo called the node. Ciliated cells in the embryonic node establish a fluid flow that is essential for normal left-right asymmetry, and disruption of the flow (or failure of other cilia to respond to flow) leads to disruption of the asymmetry. ${ }^{15-17}$

Of course, defective motility of airway epithelial cells and sperm lead to the other most noticeable symptoms associated with PCD, but several other disorders also characterize the syndrome. Some PCD patients exhibit hydrocephalus because of defective functioning of ependymal cell cilia; some exhibit anosmia, due to defects in the cilia on olfactory epithelial cells; some females are sterile because of defective cilia on the oviduct epithelium; and a small number of patients exhibit retinitis pigmentosa, presumably related to malfunctioning of rod outer segments. As always, things are not as simple as they seem, and some PCD patients also exhibit polycystic kidney disease (PKD) (see Ibanez-Tallon et $a 1^{18}$ and Saeki et $\left.a l^{19}\right)$. Until recently, the connection between cilia and PKD was a mystery. It was the discovery of the mechanism of assembly of Chlamydomonas flagella along with identification of the genes that encode the assembly machinery that provided key clues that led to the current renaissance of interest in cilia and the establishment of their essential role in many diseases (reviewed in Pazour and Rosenbaum, ${ }^{5}$ Rosenbaum and Witman, ${ }^{20}$ Ibanez-Tallon et $a l^{18}$ and Snell et $a 1^{21}$ ). Because cilia are present on most cell types, it has become clear that mutations in genes that function in cilia can lead to disorders in multiple tissues (Table 1).

\section{Assembling a cilium: the intraflagellar transport machinery}

Just over a decade ago, Joel Rosenbaum and his colleagues discovered particles rapidly $(2-4 \mu \mathrm{m} / \mathrm{s})$ moving up and down within the flagella of Chlamydomonas ${ }^{22}$ in a process they named intraflagellar transport (IFT; for recent reviews, see Rosenbaum and Witman ${ }^{20}$ and Scholey $^{23}$ ). Since then, in a flurry of activity, cell biologists have built upon this original observation to arrive at the current model for the mechanism of assembly of cilia. Since these organelles do not contain any protein biosynthetic machinery, all of the components of cilia are synthesized in the cell body and transported into the cilium. Some of the larger complexes, such as radial spokes, are partially assembled in the cell body cytoplasm. ${ }^{24}$ Flagellar components flow into the flagellum between the basal body and the plasma membrane at the base of the flagellum through a region termed the flagellar pore. ${ }^{20}$ As axonemal components assemble on the distal tip of the centriole and then onto the tip of the lengthening axoneme, new membrane and flagellar matrix (cytoplasm) move to the organelle, and the flagellum lengthens. ${ }^{25}$

The flagellar components are transported by the IFT machinery, which comprise large protein complexes called IFT particles and at least two molecular motors. IFT particles contain 17 highly conserved proteins. ${ }^{26}$ (It was large aggregates of IFT particles that Rosenbaum's group saw moving in the flagellum in 1993.) The plus-end-directed microtubule motor protein kinesin-II is essential for movement of particles and their cargo toward the tip (anterograde transport) of the flagellum, and a cytoplasmic dynein carries IFT particles back to the cell body (retrograde transport). Thus, IFT particles function as constantly moving molecular trucks on a closed loop. They travel on the microtubule doublets of the ciliary/flagellar axoneme, microtubule motors power them, and the individual structural components (eg, microtubule subunits, dynein arms, and radial spoke proteins) of the cilium/ flagellum are their cargo. ${ }^{24,27,28}$ With only a few exceptions, this IFT system discovered in Chlamydomonas is conserved in all eucaryotes that possess cilia or flagella. ${ }^{20,23}$

Of course, a system as complex as IFT and flagellar assembly/disassembly must be regulated. For example, IFT and movement of flagellar components do not stop when the flagellum reaches full length, however. The axonemal components are in constant flux, with addition and removal of proteins occurring constitutively at the tips of full-length flagella. We are only just beginning to learn how cells control flagellar length or how they determine whether or not cilia and flagella even will be formed. New studies on Chlamydomonas indicate that homologues of signaling proteins and proteins involved in the control of the mitotic spindle apparatus are key players in controlling assembly/ disassembly of flagella and flagellar length. ${ }^{29-32}$ For example, Pedersen et al ${ }^{33}$ showed that the Chlamydomonas protein CrEb1 is present at the tips of fulllength, growing, and shortening flagella, and also associates with the proximal ends of the basal bodies. EB1 family members in other organisms 
associate with the tips of cytoplasmic and spindle microtubules and are linked to microtubule assembly. ${ }^{34}$

Paralogues of other proteins related to cell division have also evolved to play roles in cilia. In studies of a long flagella mutant of Chlamydomonas, Lefebvre's group showed that a novel member of the MAP kinase superfamily of protein kinases is essential for specification of flagellar length, probably by activating flagellar shortening. ${ }^{29}$ The products of several other long flagella mutant genes may be involved in monitoring flagellar length and signaling the need to restore preset length. ${ }^{7,29,30,32,35}$ More recently, Pan et $a l^{31}$ reported that a Chlamydomonas member of the aurora protein kinase family regulates flagellar assembly/disassembly, probably through a mechanism that interacts with but is distinct from the length-regulating pathway. Auroras, which have been termed mitotic kinases, associate with centrosomes, kinetochores, and cytoplasmic microtubules in many organisms, and regulate several microtubule-dependent processes during cell division (for a recent review, see Ducat and Zheng ${ }^{36}$ ). Pan et al showed that the Chlamydomonas aurora was essential for the regulated disassembly of flagella that occurs when cells are exposed to altered ionic conditions. They proposed that the aurora is a cytoplasmic effector of disassembly in a checkpoint system that monitors and responds to environmental and developmental cues. Although a direct molecular connection has not been established between regulation of flagellar length and IFT or between initiation of flagellar assembly/disassembly and IFT, it will be surprising indeed if elements of the IFT machinery are not targets of the length-regulating MAP kinase ${ }^{29}$ or of the disassembly-regulating aurora kinase. ${ }^{31}$

\section{A ciliary synthesis: linking IFT, PKD, and sensory transduction}

Although Chlamydomonas continues to be an excellent model system for elucidating ciliary assembly mechanisms and providing new ideas on IFT and the properties of cilia and flagella, studies on the cilia of the nematode worm Caenorhabditis elegans, ${ }^{23}$ Drosophila, ${ }^{37-39}$ Tetrahymena, ${ }^{40}$ zebrafish, ${ }^{41,42}$ and mouse ${ }^{43}$ also are making fundamental contributions (reviewed in Rosenbaum and Wit$\operatorname{man}^{20}$ and Scholey ${ }^{23}$ ). The discovery of IFT ultimately led, albeit through a somewhat circuitous route, to a partial solution to the riddle of why some PCD patients exhibited polycystic kidney disorders and to a fuller appreciation of the role of cilia in sensory transduction events beyond those of light, sound, and odorant perception. A connection between the IFT story, PKD, and sensory transduction in multicellular organisms began to emerge with the discovery that chemosensory mutants in $C$. elegans, which were defective in their ability to form sensory cilia, contained mutations in genes encoding proteins of the IFT system. ${ }^{44}$ At around the same time, another group of $C$. elegans workers discovered that worm homologues (lov-1 and pkd-2) of human genes (PKD1 and PKD2) were essential for male mating behavior and localized to cilia ${ }^{45}$

In 2000, Pazour et $a l^{46}$ made the groundbreaking observation that disrupting expression of the mouse homologue (polaris/Tg737) of the Chlamydomonas IFT particle protein IFT88 led to short renal cilia and a mouse form of PKD. Finally, using the Cre/lox method to specifically disrupt a subunit of kinesin-II in kidney cells, Igarashi's group found that kidney epithelial cells in the transgenic, kinesin-II defective mouse failed to assemble cilia and developed PKD. ${ }^{47}$ (We should note that, as testament to the key role of cilia in development, null mutations in IFT genes cause embryonic lethality in the mouse.) These studies showing that the PKD genes were in worm cilia coupled with the observation that mice that failed to form normal cilia developed PKD brought into sharp focus the possibility that human PKD was a disease of cilia. As will be described below, an exciting surge in studies of the cell biology of PKD places most of the protein products of human PKD genes at the cilia. Surprisingly, none of the human PKD genes are part of the axoneme or the IFT machinery. Rather, they function in kidney development and homeostasis and are key elements in a cilium-generated signaling pathway for mechanosensation.

\section{Polycystic kidney disease}

PKD is the most common inherited disease in the United States. Current estimates are that 600000 patients have PKD in the US, with 12.5 million worldwide cases (for a recent, comprehensive review on $\mathrm{PKD}$, see Wilson ${ }^{48}$ ). The inherited PKD's include autosomal dominant (ADPKD), autosomal recessive (ARPKD), and nephronophthisis (Table 2). ADPKD, which is the most common form, occurs in 1 among 600-800 live-births and affects $\sim 500000$ persons in the US. The disease occurs during adult life and is characterized by extensive cystic enlargement of both kidneys. Of the two types of ADPKD, type I is caused by a mutation in the PKD1 gene, and type II by a mutation in the PKD2 gene. The proteins encoded by both genes are transmembrane proteins. Polycystin 1 is proposed to be a cell-cell and cellmatrix adhesion receptor ${ }^{49}$ and polycystin 2 is thought to act as a calcium-permeable membrane channel $^{50}$ (for reviews, see Boletta ${ }^{51}$ and Igarashi ${ }^{52}$ ). Autosomal recessive (ARPKD), which is an infantile form with an incidence of 1 in 20000 live-births, often leads to fetal or neonatal death due to enlargement of the kidneys. The gene disrupted in ARPKD patients, PKHD1, encodes a protein named fibrocystin or polyductin, which is a large, 4074 amino-acid polypeptide with a single transmem- 
Table 2 Cilia and PKD

\begin{tabular}{|c|c|c|c|}
\hline Gene/protein & Properties/functions & Mammals (worm, fly) & Chlamydomonas \\
\hline PKD1/polycystin 1 & $\begin{array}{l}\text { Putative receptor; complexes with } \\
\text { polycystin } 2\end{array}$ & $\begin{array}{l}\text { Primary cilia of cultured kidney } \\
\text { cells; plasma membrane (worm } \\
\text { cilia) }\end{array}$ & Not found \\
\hline PKD2/polycystin 2 & $\begin{array}{l}\text { Calcium-permeable membrane } \\
\text { channel; complexes with } \\
\text { polycystin } 1\end{array}$ & $\begin{array}{l}\text { Primary cilia \& plasma membrane } \\
\text { cultured kidney cells (worm cilia; } \\
\text { fly sperm flagellar tip: mutant } \\
\text { sperm fail to locate egg) }\end{array}$ & C_590099a \\
\hline PKHD1/fibrocystin, polyductin & Transmembrane protein & $\begin{array}{l}\text { cilia, basal body, plasma membrane } \\
\text { cultured kidney cells; kidney, } \\
\text { pancreas, liver, testis }\end{array}$ & $\begin{array}{l}\text { C_4 } 470069 \\
\text { C_-150093 } \\
\text { C_420012 }\end{array}$ \\
\hline NPHP1/nephrocystin & $\begin{array}{l}\text { Cytoplasmic protein with SH3 } \\
\text { domain; interacts with BCAR1, } \\
\text { PTK2B, tensin, NPHP2, 3,4 in } \\
\text { embryonic kidney \& testis in } \\
\text { mouse }\end{array}$ & Renal and respiratory cilia & Not found \\
\hline NPHP2/inversin & $\begin{array}{l}\text { Interacts with APC2, } \\
\text { calmodulin, \& NPHP1 }\end{array}$ & $\begin{array}{l}9+0 \text { but not } 9+2 \text { cilia; null mutant } \\
\text { mice: situs inversus, cysts in } \\
\text { kidneys and pancreas; not essential } \\
\text { for ciliogenesis }\end{array}$ & Not found \\
\hline NPHP3/nephrocystin 3 & Interacts with NPHP1 & $\begin{array}{l}\text { Embryonic node, kidney, retina, } \\
\text { respiratory epithelium, liver, biliary } \\
\text { tract and neural tissues (interaction } \\
\text { with NPHP1 suggests presence in } \\
\text { cilia) }\end{array}$ & Not found \\
\hline $\begin{array}{l}\text { NPHP4/nephrocystin } 4, \\
\text { nephroretinin }\end{array}$ & Interacts with NPHP1 & $\begin{array}{l}\text { NPHP1 interaction suggests cilia } \\
\text { location }\end{array}$ & C_380124 \\
\hline
\end{tabular}

${ }^{\mathrm{a}}$ Protein name in assembly 2.0 of the C. reinhardtii genome database available through the Department of Energy Joint Genome Institute Genome Portal (URL: http://genome.jgi-psf.org/chlre2/chlre2.home.html).

brane domain. ${ }^{53-55}$ Patients with ADPKD and ARPKD frequently have cysts in the liver and the pancreas. ${ }^{52,56}$ Familial nephronophthisis occurs as several types, appears in juveniles, adolescents, and infants, and is characterized by shrunken kidneys and renal cysts. ${ }^{57}$ To date, the following four nephronophthisis genes have been identified: NPHP1, which encodes nephrocystin; ${ }^{58,59}$ NPHP2, which encodes inversin; ${ }^{60}$ NPHP3, encoding nephrocystin $3 ;^{61}$ and NPHP4 encoding nephrocystin 4 or nephroretinin. ${ }^{62,63}$

\section{The human PKD genes are in cilia and at least two are essential for sensing flow}

Based on studies in humans and mice, almost all of the proteins disrupted in human PKDs are present on cilia (Table 2) including polycystin $1,{ }^{64,65}$ polycystin $2,{ }^{64,65}$ fibrocystin, ${ }^{66-68}$ nephrocystin, ${ }^{60}$ and inversin. ${ }^{69,60,70}$ Although nephrocystin 3 and nephrocystin 4 have not been localized to cilia, nephrocystin 4 forms a complex with nephrocystin, suggesting that they may be colocalized in cilia. ${ }^{63}$ Disruption of several additional genes in mouse also leads to PKD, and the protein products of at least two, cystin ${ }^{65,71}$ and polaris/Tg737,, ${ }^{65,72-74}$ are localized to cilia.

Interestingly, none of the human PKD genes is associated with assembly or motility of cilia. Rather, PKD patients form cilia that are normal in appear- ance, but are missing proteins that appear to be involved in sensory transduction. Polycystin 1 and polycystin 2 interact with each other ${ }^{75}$ and other proteins, ${ }^{76}$ and, although they are present in other parts of the cell, both are present in cilia (see above). In their nonciliary sites, they are proposed to function as a ligand-inducible receptor-channel complex, although no ligands have yet been identified. Furthermore, compelling evidence indicates that they are elements in a cilium-based mechanosensory system that monitors and responds to fluid flow over the surface of the renal epithelial cell (reviewed in Praetorius and Spring ${ }^{77}$ ). In elegant studies on MDCK cells in culture, Praetorius and Spring showed that bending of the $5-8 \mu \mathrm{m}$ long single cilium on the cells, either by a micropipette or by increasing the perfusate flow rate, led to a striking increase in the intracellular $\mathrm{Ca}^{2+} .{ }^{78,79}$ Moreover, the increase in intracellular $\mathrm{Ca}^{2+}$ was absolutely dependent on the presence of a cilium. ${ }^{80}$ Nauli et $a l^{64}$ showed that embryonic kidney cells isolated from a transgenic mouse that lacked functional polycystin 1 formed cilia of wild-type length, but showed no $\mathrm{Ca}^{2+}$ response to fluid shear stress. And, expression of human PKD1 in MDCK cells slowed their growth and induced them to form branching tubules in culture, rather than simple cysts. ${ }^{81}$ Thus, in normal kidneys, the renal cilia are sensory transducers in a cilium-generated signaling pathway, and polycystin 1 and polycystin 2 are key elements in that pathway. 
Understanding how disruption of ciliary function leads to PKD and to the accompanying cystic disorders in other tissues such as liver and pancreas continues to be challenging. In part, this is because we do not yet know the function of fibrocystin (the protein encoded by the autosomal recessive PKD gene) or of any of the four nephronopthisis genes. Nephrocystin 4 in mouse has been linked to signal transduction proteins, such as protein tyrosine kinases, ${ }^{82,83}$ but whether this interaction occurs in cilia and how the interaction relates to its function is unknown. Several of the PKD gene products are also associated with other cilia-related disorders. For example, all four nephronophthisis loci are linked with retinitis pigmentosa in Senior-Loken syndrome. ${ }^{62}$ Furthermore, that disruption of inversin (the nephronophthisis 2 gene product) (reviewed in Eley et $a l^{84}$ ) or PKD2 in mice ${ }^{84,85}$ causes situs inversus and that the Drosophila homologue of polycystin 2 is localized at the tip of sperm flagella and is essential for directional movement ${ }^{86}$ are compelling evidence that these and possibly all of the PKD proteins carry out as yet unidentified functions that are specific to cilia.

Confounding the attempts to understand how defects in cilia bring about PKD, almost all of the human PKD proteins are not uniquely expressed in cilia (reviewed in Igarashi and Somlo, ${ }^{52}$ Wilson, ${ }^{48}$ and Gresh et $a{ }^{87}$ ). Thus, even though defects in ciliary function are a proximal cause of the disease, the absence of the PKD proteins at other sites in the cell might also play an essential role in disease progression. Most current models about the origins of PKD incorporate the idea that the inability of the epithelial cells to detect flow leads to alterations in $\mathrm{Ca}^{2+}$ metabolism, changes in the way that cells respond to other second messengers including cAMP, and a fundamental disruption in the balance between apoptosis and proliferation of epithelial cells (reviewed in Igarashi and Somlo, ${ }^{52}$ Wilson, ${ }^{48}$ and Gresh et $a l^{87}$ ). Not to be ignored, however, is the connection between cilia, centrioles, and cell division mentioned above. Some of the PKD proteins are also localized to the basal body. ${ }^{66-68,88}$ Since centrioles are the defining unit of both basal bodies and centrosomes, malfunction of proteins associated with centrioles could interfere with their centrosomal function and lead to alterations in the cell cycle and cell proliferation. ${ }^{2,9,89,90}$

We know that disruption in the function of the PKD proteins can lead to aberrant regulation of cell proliferation/apoptosis in developing and adult tissues (reviewed in Boletta and Germino ${ }^{51}$ ), but what is the function of flow-regulated polycystin 1 and polycystin 2 signaling in a normal kidney? Recent studies show that a proteolytic, cytoplasmic fragment of polycystin 1 regulates branching morphogenesis of kidney tubule cells, suggesting that the proteins are involved in early embryonic development of the nephron or in maintenance of its normal tubular structure. ${ }^{91}$ An exciting new study shows that cessation of flow in kidney tubules of adult mice induces cleavage of the C-terminal tail of polycystin 1, followed by translocation of the fragment into the nucleus where it initiates signaling processes. ${ }^{92}$ Even though such a pathway could regulate proliferation, it is likely that polycystinmediated, flow-regulated $\mathrm{Ca}^{2+}$ signaling also influences other properties of nephron function besides cellular proliferation. For example, flow-induced signals generated within renal cilia could provide a mechanism for coupling flow to rates of ion reabsorption, or to other cellular properties related to luminal contents. ${ }^{51}$ Thus, in the embryo, the PKD genes might regulate nephron morphogenesis. In the adult, they might regulate ion and/or fluid movement across the epithelium. Defective expression of the proteins, as is seen in PKD, could bring about alterations in signaling pathways not normally related to flow-regulated pathways, and permanent changes in cellular properties that lead to overproliferation.

\section{Cilia and the obesity disorder Bardet-Biedl syndrome}

Cilia have also become scene-stealers in another human disorder, Bardet-Biedl syndrome (BBS). BBS is a rare genetic disorder characterized by obesity, polydactyly, renal abnormalities, retinal dystrophy, anosmia, situs inversus, and, in some patients, increased incidence of hypertension and diabetes (for a recent review, see Katsanis ${ }^{93}$ ). To date, eight different loci have been linked to this heterogeneous disease (Table 3). Since obesity and several of the other phenotypes that accompany BBS are common, studying this disorder potentially will yield new insights into the cellular and molecular mechanisms that underlie these important phenotypes. That several of the disease phenotypes (retinal dystrophy, situs inversus, and anosmia) already were linked to cilia suggested a connection between BBS and cilia, and several recent studies have confirmed this idea $^{94-97}$ (reviewed in Mykytyn and Sheffield ${ }^{98}$ ). The BBS1 and BBS4 gene products are essential for formation of normal cilia in mouse olfactory epithelium. ${ }^{97}$ Interestingly, $B B S 4$ knockout mice also undergo apoptotic retinal degeneration and males are sterile because their sperm fail to form a flagellum. ${ }^{95,96}$ On the other hand, the $B B S 4$ mice (which exhibit anosmia) show normal motile cilia in the airway and respiratory epithelial, as well as cilia of normal appearance in the renal epithelium. Thus, BBS4 protein is not globally required for cilia formation.

Investigators turned to Chlamydomonas to identify the BBS5 gene. Using a novel, subtractive bioinformatics approach based on the Chlamydomonas genome, Li et al ${ }^{99}$ determined that a flagellaand basal body-specific gene was linked to the BBS5 locus. Further analysis showed that the novel 
Table 3 Cilia and BBS

\begin{tabular}{|c|c|c|c|c|}
\hline \multirow[t]{2}{*}{ Gene } & \multirow[t]{2}{*}{ Protein property/function } & \multicolumn{3}{|c|}{ Location } \\
\hline & & Mammals & Worm/fly & Chlamydomonas \\
\hline BBS1 & Unknown function & $\begin{array}{l}\text { Null mutant mice: no olfactory } \\
\text { cilia but normal respiratory and } \\
\text { renal cilia }\end{array}$ & Basal body and cilia in worm & C_1020033 \\
\hline BBS2 & Unknown function & No information & Basal body and cilia in worm & C_200109 \\
\hline$B B S 3$ & $\begin{array}{l}\text { Small G-protein } \\
\text { Transport along ciliary axoneme }\end{array}$ & No information & Basal body and cilia in worm & C_970056 \\
\hline$B B S 4$ & $\begin{array}{l}\text { Target/anchor pericentriolar } \\
\text { protein PCM1 }\end{array}$ & $\begin{array}{l}\text { Basal body and centrosomes } \\
\text { Loss results in disorganized } \\
\text { microtubules, mislocalization of } \\
\text { centriolar proteins, failure of cell } \\
\text { division } \\
\text { Null mutant mice: no olfactory } \\
\text { cilia but normal respiratory and } \\
\text { renal cilia }\end{array}$ & No information & C_70158 \\
\hline BBS5 & Generation of cilia and flagella & Basal body & Basal body in worm & $\begin{array}{l}\text { C_270058 } \\
\text { RNAi knockdown } \\
\text { cells aflagellate }\end{array}$ \\
\hline BBS6 & Putative chaperonin & No information & $\begin{array}{l}\text { No information } \\
\text { Basal body and cilia in worm }\end{array}$ & Not found \\
\hline$B B S 7$ & $\begin{array}{l}\text { Bidirectional movement along } \\
\text { ciliary axoneme }\end{array}$ & Basal body & $\begin{array}{l}\text { Compromised IFT, shortened } \\
\text { cilia in worm mutant }\end{array}$ & C_1330001 \\
\hline BBS8 & 505aa; TPR domain & $\begin{array}{l}\text { Centrosome, Basal body, cilia } \\
\text { (retina, olfactory neurons, } \\
\text { spermatids, bronchial epithelial } \\
\text { cells) }\end{array}$ & Basal body and cilia in worm & C_970071 \\
\hline & Associates with PCM1 & & $\begin{array}{l}\text { Loss of BBS8 protein in worm } \\
\text { results in compromised IFT, } \\
\text { shortened cilia }\end{array}$ & \\
\hline & $\begin{array}{l}\text { Bidirectional movement along } \\
\text { ciliary axoneme }\end{array}$ & & & \\
\hline
\end{tabular}

protein encoded by this previously unidentified gene localized to basal bodies in mouse and that its disruption by RNAi in Chlamydomonas generated cells that were aflagellate and showed cleavage furrow defects. BBS8 protein also localizes to ciliated structures such as the connecting cilium of the retina and columnar, ciliated epithelial cells in the lung. ${ }^{94}$ Moreover, like BBS4, BBS8 localizes to centrosomes and basal bodies. Although studies on mouse and Chlamydomonas did not yield information about BBS2 or BBS7, experiments in C. elegans place them at the cilia. ${ }^{94}$ Worm BBS2 is expressed only in ciliated cells, probably in association with basal bodies. ${ }^{94}$ Surprisingly, the worm BBS7 homologue moves bidirectionally along the ciliary axoneme and is required for the assembly/function of IFT particles, implicating BBS7 in intraflagellar transport. ${ }^{100}$ One of the more interesting ideas to emerge from studies of the BBS proteins in Chlamydomonas, C. elegans, mouse, and humans is that those that associate with centrioles may play roles both in ciliary assembly/function and in cell division. In their centriolar role as basal bodies, they influence cilia function, and in their centriolar role in centrosomes, they influence cell division.

\section{Cilium-generated signaling: a direct role for IFT in sensory transduction during Hedgehog signaling in mouse and fertilization in Chlamydomonas}

The relationship between the BBS proteins and several of the BBS phenotypes remains mysterious. For example, why should interference with a basal body protein lead to obesity or diabetes? One speculative idea is that cilia are required for the proper function of as yet uncharacterized receptors involved in these phenotypes. In other words, unique properties of cilia might favor their widespread use as signal generators. With the exception of olfactory epithelial cilia and rod outer segments, the ciliary sensory functions described so far in this review are involved in mechanosensation. Several receptors, however, with known extracellular ligands also are present on cilia. Integrins have been localized to the primary cilium of MDCK cells, where they are proposed to influence intracellular $\mathrm{Ca}^{2+}$ levels in a flow-independent mechanism. ${ }^{101}$ Furthermore, somatostatin receptor $3^{102}$ and the serotonin receptor $5-\mathrm{Ht}_{6}{ }_{6}{ }^{103}$ are localized to neuronal cilia in the brain. Thus, it does not require too much of a leap to speculate that other membrane 
receptors-for example, those involved in the sensation of fullness-are localized on cilia, and could be involved in regulating food intake and overall energy metabolism. In fact, many brain neuronal cells that are responsive to the weightregulating protein leptin display somatostatin 3 receptors on their neuronal cilia. ${ }^{104}$ Moreover, recent results from studies of Sonic Hedgehog signaling suggest that elements of this important developmental pathway require cilia. Huangfu et $a l^{105}$ reported that the Hedgehog signaling pathway was disrupted in mouse embryos with lesions in kinesinII and two IFT particle proteins. Of course, it is possible that these IFT machinery proteins play a nonciliary role in the pathway, but one explanation for the results is that Hedgehog pathway proteins require cilia for their function.

Many of the unique properties of cilia could have led to their exploitation during evolution as sensory transducers. Because they project a cell-type specific distance from the cell body, they are perfectly designed probes (cybernetic probes, ${ }^{106}$ cellular antennae ${ }^{107}$ ) of the external milieu. The ciliary membrane and the cytoplasmic contents of the organelle are well isolated from the cell body, offering all of the advantages of compartmentalization. Moreover, the IFT machinery that assembles them makes it possible to transport proteins in a rapid, regulated manner between the cilia and the cell body. And, the IFT machinery seems exploitable for use directly in signaling pathways.

Chlamydomonas might again point the way to a deeper understanding of how cells use their cilia. In recent studies on Chlamydomonas, we have shown that flagellar adhesion-activated sensory transduction during fertilization ${ }^{108}$ requires the IFT machinery. ${ }^{109}$ The flagellar adhesion molecule agglutinin of Chlamydomonas is present both on the flagella and cell body of gametes. Surprisingly, and for as yet unknown reasons, the agglutinin functions as an adhesion and signaling molecule only when it is present on the flagella. The cell body form is nonfunctional (reviewed in Pan et $a l^{110}$ ). Moreover, the ability of the flagellar-localized adhesion molecules to activate the signaling pathway during cellcell adhesion requires that the IFT machinery be active. ${ }^{109}$ Existing, intact flagella in which IFT has been rendered inactive by a temperature-sensitive mutation are able to undergo flagellar adhesion that is indistinguishable from the adhesion of wild-type gametes. That is, the agglutinins are functioning normally in their role as adhesion molecules in the absence of IFT. Adhesion in the IFT defective flagella, however, fails to activate the cilium-generated signaling pathway that leads to gamete fusion. ${ }^{109}$ Moreover, the first biochemically detectable flagellar response to flagellar adhesion, activation of a protein tyrosine kinase, did not occur when IFT was inactivated. ${ }^{111}$ Thus, in intact flagella, IFT is essential for coupling interactions between the flagellar adhesion molecules on gametes of the opposite sex to activation of intracellular signaling. It will be surprising if cilia of multicellular organisms have not taken similar advantage of the IFT machinery to accomplish cilium-generated signaling during sensory transduction.

\section{Concluding remarks}

A remarkable interplay of recent studies on disparate organisms, including the unicellular green alga Chlamydomonas and the nematode worm C. elegans, has yielded fundamental insights into the roles of cilia and flagella in human health and disease. Emerging from this flurry of activity in ciliary biology has come the concept that the primary cilium is a mechanosensor in renal epithelial cells and that disruption of the mechanosensing properties of the cilium leads to cellular overproliferation. Dissecting the cellular pathways that link this seemingly innocuous organelle to the cell cycle of renal epithelial cells will be challenging, but promises to be fruitful for understanding PKD. Even more mysterious is why defects in basal bodies/cilia should lead to an obesity disorder. Dissecting the role of cilia in this intriguing disorder presents even more challenges, but also offers the possibility for exciting new insights into disorders with profound health consequences in North America. Finally, Chlamydomonas may again lead the way to new discoveries on the mechanisms of cilium-generated signaling as cell biologists learn more about the direct role that IFT plays during sensory transduction in this organism.

\section{References}

1 Wheatley DN. Primary cilia in normal and pathological tissues. Pathobiology 1995;63:222-238.

2 Wheatley DN, Wang AM, Strugnell GE. Expression of primary cilia in mammalian cells. Cell Biol Int 1996;20:73-81.

3 Praetorius HA, Spring KR. A physiological view of the primary cilium. Annu Rev Physiol 2004.

4 Corey DP, Garcia-Anoveros J, Holt JR, et al. TRPA1 is a candidate for the mechanosensitive transduction channel of vertebrate hair cells. Nature 2004;432: 723-730.

5 Pazour GJ, Rosenbaum JL. Intraflagellar transport and cilia-dependent diseases. Trends Cell Biol 2002;12: 551-555.

6 Pazour GJ. Intraflagellar transport and cilia-dependent renal disease: the ciliary hypothesis of polycystic kidney disease. J Am Soc Nephrol 2004; 15:2528-2536.

7 Silflow CD, Lefebvre PA. Assembly and motility of eukaryotic cilia and flagella. Lessons from Chlamydomonas reinhardtii. Plant Physiol 2001;127:15001507.

8 Rieder CL, Faruki S, Khodjakov A. The centrosome in vertebrates: more than a microtubule-organizing center. Trends Cell Biol 2001;11:413-419. 
9 Beisson J, Wright M. Basal body/centriole assembly and continuity. Curr Opin Cell Biol 2003;15:96-104.

10 Dutcher SK. Elucidation of basal body and centriole functions in Chlamydomonas reinhardtii. Traffic 2003;4:443-451.

11 Afzelius BA. A human syndrome caused by immotile cilia. Science 1976;193:317-319.

12 Berdon WE, Willi U. Situs inversus, bronchiectasis, and sinusitis and its relation to immotile cilia: history of the diseases and their discoverers-Manes Kartagener and Bjorn Afzelius. Pediatr Radiol 2004; $34: 38-42$.

13 Afzelius BA. Cilia-related diseases. J Pathol 2004;204: 470-477.

14 Geremek M, Witt M. Primary ciliary dyskinesia: genes, candidate genes and chromosomal regions. J Appl Genet 2004;45:347-361.

15 McGrath J, Brueckner M. Cilia are at the heart of vertebrate left-right asymmetry. Curr Opin Genet Dev 2003;13:385-392.

16 McGrath J, Somlo S, Makova S, et al. Two populations of node monocilia initiate left-right asymmetry in the mouse. Cell 2003;114:61-73.

17 Nonaka S, Shiratori H, Saijoh Y, et al. Determination of left-right patterning of the mouse embryo by artificial nodal flow. Nature 2002;418:96-99.

18 Ibanez-Tallon I, Heintz N, Omran H. To beat or not to beat: roles of cilia in development and disease. Hum Mol Genet 2003;12:R27-R35.

19 Saeki H, Kondo S, Morita T, et al. Immotile cilia syndrome associated with polycystic kidney. J Urol 1984;132:1165-1166.

20 Rosenbaum JL, Witman GB. Intraflagellar transport. Nat Rev Mol Cell Biol 2002;3:813-825.

21 Snell WJ, Pan J, Wang Q. Cilia and flagella revealed: from flagellar assembly in Chlamydomonas to human obesity disorders. Cell 2004;117:693-697.

22 Kozminski KG, Johnson KA, Forscher P, et al. A motility in the eukaryotic flagellum unrelated to flagellar beating. Proc Natl Acad Sci USA 1993;90:5519-5523.

23 Scholey JM. Intraflagellar transport. Annu Rev Cell Dev Biol 2003;19:423-443.

24 Qin H, Diener DR, Geimer S, et al. Intraflagellar transport (IFT) cargo: IFT transports flagellar precursors to the tip and turnover products to the cell body. J Cell Biol 2004;164:255-266.

25 Marshall WF, Rosenbaum JL. Intraflagellar transport balances continuous turnover of outer doublet microtubules: implications for flagellar length control. J Cell Biol 2001;155:405-414.

26 Cole DG. The intraflagellar transport machinery of Chlamydomonas reinhardtii. Traffic 2003;4:435-442.

27 Piperno G, Mead K, Henderson S. Inner dynein arms but not outer dynein arms require the activity of kinesin homologue protein KHP1(FLA10) to reach the distal part of flagella in Chlamydomonas. J Cell Biol 1996;133:371-379.

28 Qin H, Rosenbaum JL, Barr MM. An autosomal recessive polycystic kidney disease gene homolog is involved in intraflagellar transport in $C$. elegans ciliated sensory neurons. Curr Biol 2001;11:457-461.

29 Berman SA, Wilson NF, Haas NA, et al. A novel MAP kinase regulates flagellar length in Chlamydomonas. Curr Biol 2003;13:1145-1149.

30 Tam LW, Dentler WL, Lefebvre PA. Defective flagellar assembly and length regulation in LF3 null mutants in Chlamydomonas. J Cell Biol 2003;163:597-607.
31 Pan J, Wang Q, Snell WJ. An aurora kinase is essential for flagellar disassembly in Chlamydomonas. Dev Cell 2004;6:445-451.

32 Wilson NF, Lefebvre PA. Regulation of flagellar assembly by glycogen synthase kinase 3 in Chlamydomonas reinhardtii. Eukaryot Cell 2004;3:13071319.

33 Pedersen LB, Geimer S, Sloboda RD, et al. The microtubule plus end-tracking protein EB1 is localized to the flagellar tip and basal bodies in Chlamydomonas reinhardtii. Curr Biol 2003;13: 1969-1974

34 Tirnauer JS, Bierer BE. EB1 proteins regulate microtubule dynamics, cell polarity, and chromosome stability. J Cell Biol 2000;149:761-766.

35 Nguyen RL, Tam LW, Lefebvre PA. The LF1 Gene of Chlamydomonas reinhardtii encodes a novel protein required for flagellar length control. Genetics 2004.

36 Ducat D, Zheng Y. Aurora kinases in spindle assembly and chromosome segregation. Exp Cell Res 2004;301:60-67.

37 Sarpal R, Todi SV, Sivan-Loukianova E, et al. Drosophila KAP interacts with the kinesin II motor subunit KLP64D to assemble chordotonal sensory cilia, but not sperm tails. Curr Biol 2003;13:1687-1696.

38 Han YG, Kwok BH, Kernan MJ. Intraflagellar transport is required in Drosophila to differentiate sensory cilia but not sperm. Curr Biol 2003;13:1679-1686.

39 Witman GB. Cell motility: deaf Drosophila keep the beat. Curr Biol 2003;13:R796-R798.

40 Brown JM, Fine NA, Pandiyan G, et al. Hypoxia regulates assembly of cilia in suppressors of Tetrahymena lacking an intraflagellar transport subunit gene. Mol Biol Cell 2003;14:3192-3207.

41 Sun Z, Amsterdam A, Pazour GJ, et al. A genetic screen in zebrafish identifies cilia genes as a principal cause of cystic kidney. Development 2004;131:40854093.

42 Tsujikawa M, Malicki J. Intraflagellar transport genes are essential for differentiation and survival of vertebrate sensory neurons. Neuron 2004;42:703-716.

43 Guay-Woodford LM. Murine models of polycystic kidney disease: molecular and therapeutic insights. Am J Physiol Renal Physiol 2003;285:F1034-F1049.

44 Cole DG, Diener DR, Himelblau AL, et al. Chlamydomonas kinesin-II-dependent intraflagellar transport (IFT): IFT particles contain proteins required for ciliary assembly in Caenorhabditis elegans sensory neurons. J Cell Biol 1998;141:993-1008.

45 Barr MM, Sternberg PW. A polycystic kidney-disease gene homologue required for male mating behaviour in C. elegans. Nature 1999;401:386-389.

46 Pazour GJ, Dickert BL, Vucica Y, et al. Chlamydomonas IFT88 and its mouse homologue, polycystic kidney disease gene $\operatorname{tg} 737$, are required for assembly of cilia and flagella. J Cell Biol 2000;151:709-718.

47 Lin F, Hiesberger T, Cordes K, et al. Kidney-specific inactivation of the KIF3A subunit of kinesin-II inhibits renal ciliogenesis and produces polycystic kidney disease. Proc Natl Acad Sci USA 2003;100: 5286-5291.

48 Wilson PD. Polycystic kidney disease. N Engl J Med 2004;350:151-164.

49 Hughes J, Ward CJ, Peral B, et al. The polycystic kidney disease 1 (PKD1) gene encodes a novel protein with multiple cell recognition domains. Nat Genet 1995;10:151-160. 
50 Mochizuki T, Wu G, Hayashi T, et al. PKD2, a gene for polycystic kidney disease that encodes an integral membrane protein. Science 1996;272:1339-1342.

51 Boletta A, Germino GG. Role of polycystins in renal tubulogenesis. Trends Cell Biol 2003;13:484-492.

52 Igarashi P, Somlo S. Genetics and pathogenesis of polycystic kidney disease. J Am Soc Nephrol 2002;13: 2384-2398.

53 Onuchic LF, Furu L, Nagasawa Y, et al. PKHD1, the polycystic kidney and hepatic disease 1 gene, encodes a novel large protein containing multiple immuno-

globulin-like plexin-transcription-factor domains and parallel beta-helix 1 repeats. Am J Hum Genet 2002; 70:1305-1317.

54 Ward CJ, Hogan MC, Rossetti S, et al. The gene mutated in autosomal recessive polycystic kidney disease encodes a large, receptor-like protein. Nat Genet 2002;30:259-269.

55 Hiesberger T, Bai Y, Shao X, et al. Mutation of hepatocyte nuclear factor-1beta inhibits Pkhd1 gene expression and produces renal cysts in mice. J Clin Invest 2004;113:814-825.

56 Cano DA, Murcia NS, Pazour GJ, et al. Orpk mouse model of polycystic kidney disease reveals essential role of primary cilia in pancreatic tissue organization. Development 2004;131:3457-3467.

57 Hildebrandt F. Nephronopthisis-medullary cystic kidney disease. In: Avner ED, Harmon WE, Niaudet $\mathrm{P}$ (eds.) Pediatric Nephrology, 5th edn. Lipincott, Williams and Williams: Philadelphia, 2004, pp 665673.

58 Saunier S, Calado J, Heilig R, et al. A novel gene that encodes a protein with a putative src homology 3 domain is a candidate gene for familial juvenile nephronophthisis. Hum Mol Genet 1997;6:23172323.

59 Hildebrandt F, Otto E, Rensing C, et al. A novel gene encoding an $\mathrm{SH} 3$ domain protein is mutated in nephronophthisis type 1. Nat Genet 1997;17: 149-153.

60 Otto EA, Schermer B, Obara T, et al. Mutations in INVS encoding inversin cause nephronophthisis type 2, linking renal cystic disease to the function of primary cilia and left-right axis determination. Nat Genet 2003;34:413-420.

61 Olbrich H, Fliegauf M, Hoefele J, et al. Mutations in a novel gene, NPHP3, cause adolescent nephronophthisis, tapeto-retinal degeneration and hepatic fibrosis. Nat Genet 2003;34:455-459.

62 Otto E, Hoefele J, Ruf R, et al. A gene mutated in nephronophthisis and retinitis pigmentosa encodes a novel protein, nephroretinin, conserved in evolution. Am J Hum Genet 2002;71:1161-1167.

63 Mollet G, Salomon R, Gribouval O, et al. The gene mutated in juvenile nephronophthisis type 4 encodes a novel protein that interacts with nephrocystin. Nat Genet 2002;32:300-305.

64 Nauli SM, Alenghat FJ, Luo Y, et al. Polycystins 1 and 2 mediate mechanosensation in the primary cilium of kidney cells. Nat Genet 2003;33:129-137.

65 Yoder BK, Hou X, Guay-Woodford LM. The polycystic kidney disease proteins, polycystin-1, polycystin-2, polaris, and cystin, are co-localized in renal cilia. J Am Soc Nephrol 2002;13:2508-2516.

66 Ward CJ, Yuan D, Masyuk TV, et al. Cellular and subcellular localization of the ARPKD protein; fibro- cystin is expressed on primary cilia. Hum Mol Genet 2003;12:2703-2710.

67 Wang S, Luo Y, Wilson PD, et al. The autosomal recessive polycystic kidney disease protein is localized to primary cilia, with concentration in the basal body area. J Am Soc Nephrol 2004;15:592-602.

68 Zhang MZ, Mai W, Li C, et al. PKHD1 protein encoded by the gene for autosomal recessive polycystic kidney disease associates with basal bodies and primary cilia in renal epithelial cells. Proc Natl Acad Sci USA 2004;101:2311-2316.

69 Morgan D, Eley L, Sayer J, et al. Expression analyses and interaction with the anaphase promoting complex protein Apc2 suggest a role for inversin in primary cilia and involvement in the cell cycle. Hum Mol Genet 2002;11:3345-3350.

70 Watanabe D, Saijoh Y, Nonaka S, et al. The left-right determinant Inversin is a component of node monocilia and other 9+0 cilia. Development 2003;130: 1725-1734.

71 Hou X, Mrug M, Yoder BK, et al. Cystin, a novel ciliaassociated protein, is disrupted in the cpk mouse model of polycystic kidney disease. J Clin Invest 2002;109:533-540.

72 Yoder BK, Tousson A, Millican L, et al. Polaris, a protein disrupted in orpk mutant mice, is required for assembly of renal cilium. Am J Physiol Renal Physiol 2002;282:F541-F552.

73 Brown NE, Murcia NS. Delayed cystogenesis and increased ciliogenesis associated with the re-expression of polaris in Tg737 mutant mice. Kidney Int 2003;63:1220-1229.

74 Taulman PD, Haycraft CJ, Balkovetz DF, et al. Polaris, a protein involved in left-right axis patterning, localizes to basal bodies and cilia. Mol Biol Cell 2001;12:589-599.

75 Hanaoka K, Qian F, Boletta A, et al. Co-assembly of polycystin-1 and -2 produces unique cation-permeable currents. Nature 2000;408:990-994.

76 Roitbak T, Ward CJ, Harris PC, et al. A polycystin-1 multiprotein complex is disrupted in polycystic kidney disease cells. Mol Biol Cell 2004;15:13341346.

77 Praetorius HA, Spring KR. The renal cell primary cilium functions as a flow sensor. Curr Opin Nephrol Hypertens 2003;12:517-520.

78 Praetorius HA, Spring KR. Bending the MDCK cell primary cilium increases intracellular calcium. J Membr Biol 2001;184:71-79.

79 Praetorius HA, Frokiaer J, Nielsen S, et al. Bending the primary cilium opens $\mathrm{Ca}^{2+}$-sensitive intermediateconductance $\mathrm{K}+$ channels in MDCK cells. J Membr Biol 2003;191:193-200.

80 Praetorius HA, Spring KR. Removal of the MDCK cell primary cilium abolishes flow sensing. J Membr Biol 2003;191:69-76.

81 Boletta A, Qian F, Onuchic LF, et al. Polycystin-1, the gene product of $P K D 1$, induces resistance to apoptosis and spontaneous tubulogenesis in MDCK cells. Mol Cell 2000;6:1267-1273.

82 Benzing T, Gerke P, Hopker K, et al. Nephrocystin interacts with Pyk2, p130(Cas), and tensin and triggers phosphorylation of Pyk2. Proc Natl Acad Sci USA 2001;98:9784-9789.

83 Donaldson JC, Dempsey PJ, Reddy S, et al. Crkassociated substrate p130(Cas) interacts with nephrocystin and both proteins localize to cell-cell contacts 
of polarized epithelial cells. Exp Cell Res 2000;256: 168-178.

84 Eley L, Turnpenny L, Yates LM, et al. A perspective on inversin. Cell Biol Int 2004;28:119-124.

85 Pennekamp P, Karcher C, Fischer A, et al. The ion channel polycystin-2 is required for left-right axis determination in mice. Curr Biol 2002;12:938-943.

86 Watnick TJ, Jin Y, Matunis E, et al. A flagellar polycystin-2 homolog required for male fertility in Drosophila. Curr Biol 2003;13:2179-2184.

87 Gresh L, Fischer E, Reimann A, et al. A transcriptional network in polycystic kidney disease. EMBO J 2004;23:1657-1668.

88 Menezes LF, Cai Y, Nagasawa Y, et al. Polyductin, the PKHD1 gene product, comprises isoforms expressed in plasma membrane, primary cilium, and cytoplasm. Kidney Int 2004;66:1345-1355.

89 Hagiwara H, Ohwada N, Takata K. Cell biology of normal and abnormal ciliogenesis in the ciliated epithelium. Int Rev Cytol 2004;234:101-141.

90 Watnick T, Germino G. From cilia to cyst. Nat Genet 2003;34:355-356.

91 Nickel C, Benzing T, Sellin L, et al. The polycystin-1 C-terminal fragment triggers branching morphogenesis and migration of tubular kidney epithelial cells. J Clin Invest 2002;109:481-489.

92 Chauvet V, Xin T, Husson $\mathrm{H}$, et al. Mechanical stimuli induce cleavage and nuclear translocation of the polycystin-1 C terminus. J Clin Invest 2004;114: 1433-1443.

93 Katsanis N. The oligogenic properties of Bardet-Biedl syndrome. Hum Mol Genet 2004;13:R65-R71.

94 Ansley SJ, Badano JL, Blacque OE, et al. Basal body dysfunction is a likely cause of pleiotropic BardetBiedl syndrome. Nature 2003;425:628-633.

95 Kim JC, Badano JL, Sibold S, et al. The Bardet-Biedl protein BBS4 targets cargo to the pericentriolar region and is required for microtubule anchoring and cell cycle progression. Nat Genet 2004;36:462-470.

96 Mykytyn K, Mullins RF, Andrews M, et al. BardetBiedl syndrome type 4 (BBS4)-null mice implicate Bbs4 in flagella formation but not global cilia assembly. Proc Natl Acad Sci USA 2004;101:86648669.

97 Kulaga HM, Leitch CC, Eichers ER, et al. Loss of BBS proteins causes anosmia in humans and defects in olfactory cilia structure and function in the mouse. Nat Genet 2004;36:994-998.

98 Mykytyn K, Sheffield VC. Establishing a connection between cilia and Bardet-Biedl syndrome. Trends Mol Med 2004;10:106-109.

$99 \mathrm{Li}$ JB, Gerdes JM, Haycraft CJ, et al. Comparative genomics identifies a flagellar and basal body pro- teome that includes the BBS5 human disease gene. Cell 2004;117:541-552.

100 Blacque OE, Reardon MJ, Li C, et al. Loss of C. elegans BBS-7 and BBS-8 protein function results in cilia defects and compromised intraflagellar transport. Genes Dev 2004;18:1630-1642.

101 Praetorius HA, Praetorius J, Nielsen S, et al. Beta1-integrins in the primary cilium of MDCK cells potentiate fibronectin-induced $\mathrm{Ca}^{2+}$ signaling. Am J Physiol Renal Physiol 2004;287:F969-F978.

102 Handel M, Schulz S, Stanarius A, et al. Selective targeting of somatostatin receptor 3 to neuronal cilia. Neuroscience 1999;89:909-926.

103 Brailov I, Bancila M, Brisorgueil MJ, et al. Localization of 5-HT(6) receptors at the plasma membrane of neuronal cilia in the rat brain. Brain Res 2000;872: 271-275.

104 Stepanyan Z, Kocharyan A, Pyrski M, et al. Leptintarget neurones of the rat hypothalamus express somatostatin receptors. J Neuroendocrinol 2003;15: 822-830.

105 Huangfu D, Liu A, Rakeman AS, et al. Hedgehog signalling in the mouse requires intraflagellar transport proteins. Nature 2003;426:83-87.

106 Poole CA, Flint MH, Beaumont BW. Analysis of the morphology and function of primary cilia in connective tissues: a cellular cybernetic probe? Cell Motil 1985;5:175-193.

107 Pazour GJ, Witman GB. The vertebrate primary cilium is a sensory organelle. Curr Opin Cell Biol 2003;15: 105-110.

108 Solter KM, Gibor A. Evidence for role of flagella as sensory transducers in mating of Chlamydomonas reinhardii. Nature 1977;265:444-445.

109 Pan J, Snell WJ. Kinesin-II is required for flagellar sensory transduction during fertilization in Chlamydomonas. Mol Biol Cell 2002;13:14171426.

110 Pan J, Misamore MJ, Wang Q, et al. Protein transport and signal transduction during fertilization in Chlamydomonas. Traffic 2003;4:452-459.

111 Wang Q, Snell WJ. Flagellar adhesion between mating type plus and mating type minus gametes activates a flagellar protein-tyrosine kinase during fertilization in Chlamydomonas. J Biol Chem 2003;278:3293632942.

112 Jensen CG, Poole CA, McGlashan SR, et al. Ultrastructural, tomographic and confocal imaging of the chondrocyte primary cilium in situ. Cell Biol Int 2004;28:101-110.

113 Kessel RG, Kardon RH. Tissues and Organs: A Textatlas of Scanning Electron Microscopy. WH Freeman and Company: San Francisco, CA, 1979. 